\title{
Event vertex reconstruction with deep neural networks for the DarkSide-20k experiment
}

\author{
Victor Goicoechea-Casanueva ${ }^{1, *}$, Alexander $\mathrm{Kish}^{1, * *}$, and Jelena Maricic ${ }^{1, * * *}$ \\ on behalf of the DarkSide collaboration \\ ${ }^{1}$ Department of Physics and Astronomy, University of Hawaii at Manoa, Honolulu, USA
}

\begin{abstract}
While deep learning techniques are becoming increasingly more popular in high-energy and, since recently, neutrino experiments, they are less confidently used in direct dark matter searches based on dual-phase noble gas TPCs optimized for low-energy signals from particle interactions.

In the present study, the application of modern deep learning methods for event vertex reconstruction is demonstrated with an example of the 50-tonne liquid argon DarkSide-20k TPC with 8200 photosensors.

The developed methods successfully reconstruct event positions within sub-cm precision and apply to any dual-phase argon or xenon TPC of arbitrary size with any sensor shape and array pattern.
\end{abstract}

\section{Dual-phase TPCs, Electroluminescence, and Position Reconstruction}

Particle detection and identification in a dual-phase time-projection chamber (TPC) relies on a prompt light pulse (S1) induced by scintillation, followed by a delayed pulse (S2) associated with ionization electrons. The latter are drifted upwards in an electric field and extracted into a thin layer of gas, where after being accelerated induce a secondary light signal by electroluminescence. Both S1 and S2 signals are detected by photosensors below and above the active volume.

A strong feature of a TPC particle detector is the possibility to determine all three coordinates of an interaction vertex in the target volume, which are used for position-dependent signal corrections and fiducialization of the target volume for background suppression. The $z$-coordinate is inferred with sub-mm precision from the measured time difference between $\mathrm{S} 1$ and S2 with known electron drift velocity and TPC drift length. The $X Y$ coordinates are reconstructed from the $\mathrm{S} 2$ signal distribution.

The proportional scintillation signal S2 is generated in a well-defined plane between the liquid-gas interface and the anode electrode (figure 1). About half of the light is observed by a fraction of the photosensors in the top array, just above the $\mathrm{S} 2$ production region, while the other half is rather uniformly distributed among the bottom array. The top pattern, being localized and consisting mostly of direct, non-reflected light, is used for XY vertex reconstruction (figure 1). For an optimal XY resolution, the top photosensor array is typically

\footnotetext{
*e-mail: victorgc@hawaii.edu

**e-mail: akish@phys.hawaii.edu

***e-mail: jelena@phys.hawaii.edu
} 
placed at a distance $H$ from the production region roughly equal to the size of the sensor unit $S$.

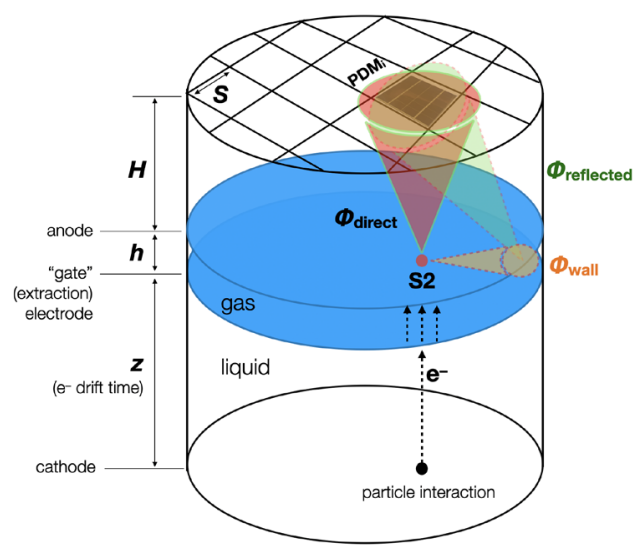

Figure 1. A simplified model (not-to-scale) of a dual-phase TPC and propagation of the electroluminescence light (S2) from the production in the gas layer to the detection by the top array of photodetection modules (PDMs) at a vertical distance $H$.

The conventional event vertex reconstruction algorithms are based on analysis of light response functions (LRFs) and likelihood fitting of the top array light patterns [1]. The hit patterns are usually generated by detailed optical Monte Carlo (MC) simulations, or can even be deduced in an accurate analysis from measured data. While likelihood-based algorithms give very good vertex reconstruction resolution (sub-cm precision [1]), they are iterative and expected to be computationally heavy and slow. Machine learning techniques were shown to perform with high accuracy and computing efficiency, for instance, in the XENON10 experiment [2].

Modern deep learning methods are being increasingly applied for event reconstruction in neutrino experiments such as MicroBooNE [3, 4] and DUNE [5, 6]. Moreover, studies based on convolution neural networks (CNNs) are also found for dual-phase TPC-based experiments, for example, the EXO-200 [7], the XENONnNT [8], and the DarkSide-50 [9] collaborations. In addition to these, several applications of various neural network techniques were demonstrated for the LUX xenon TPC [10], not covering direct XY vertex reconstruction.

The next-generation experiments based on dual-phase TPCs, which will consist of tens of tonnes of condensed argon or xenon in the target and will be equipped with thousands of photosensors [11], appear as an appealing subject for deep learning studies.

In this work, we describe the network design, training techniques, and software tools developed to train networks with the purpose of event vertex reconstruction in the DarkSide-20k experiment, with the ultimate objective of developing a complete deep neural network (DNN) based data reconstruction chain that can be adapted to any size, shape, and photosensor placement for dual-phase TPCs. DarkSide-20k will be a dual-phase argon TPC of about $50 \mathrm{t}$ target mass for dark matter detection [12] at Laboratori Nazionali del Gran Sasso (LNGS), Italy, with 8200 silicon photodetection modules in the inner detector. A future experiment ARGO is being planned at SNOLAB, Canada, which will include an argon TPC with a target mass of $370 \mathrm{t}$ [11]. A comparison with past and current TPCs for direct dark matter searches is shown in figure 2. 


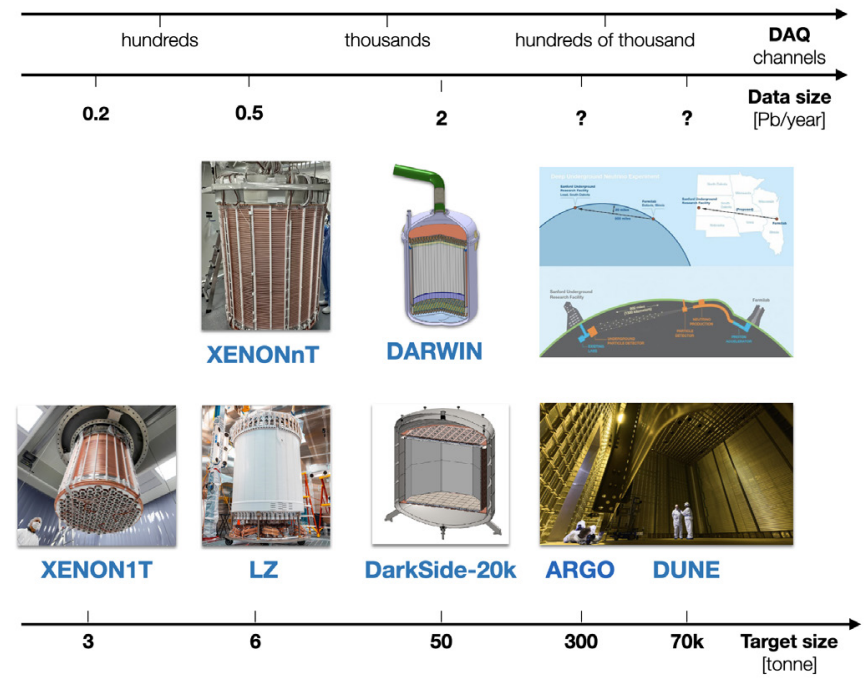

Figure 2. Approximate characteristics of dual-phase xenon and argon TPCs for direct dark matter searches (excluding DUNE): target size, number of DAQ channels, and data storage size.

Due to the radiogenic nature of a large component of the background, most background energy deposits are found in the edges of the TPC. This fact combined with the fidualization of the active volume allows to discriminate events of this nature. For this reason, an adequate and precise position reconstruction is needed. An upper bound estimate for the XY spatial resolution can be obtained from observing the amount of events of this nature that leak into the fiducial volume $(\mathrm{FV})$ given a certain spatial resolution $\left(\sigma_{r}\right)$. Figure 3 shows the spatial distribution of events of radiogenic nature as well as the percentage $(R)$ of events falling into the FV given a certain spatial resolution. These plots are based on a Monte Carlo simulation that includes properties of the materials used in the construction of the DarkSide-20k detector. It can be observed that the events start to leak into the FV when spatial resolutions reach values greater than $\sigma_{r}>1 \mathrm{~cm}$.

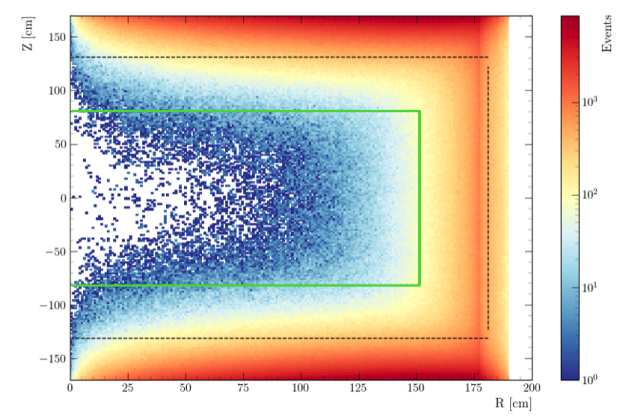

(a)

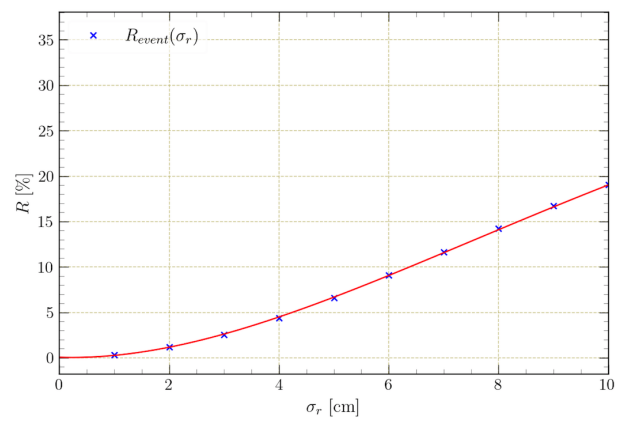

(b)

Figure 3. a) Background spatial distribution of radiogenic nature events. In green, the FV boundaries. b) Leakage of background events into the FV as a function of XY spatial resolution. 


\section{Vertex Reconstruction for the DarkSide-20k Experiment}

The data for the study have been generated in Monte Carlo simulations with G4DS [13], a GEANT4-based application that includes a detailed model of the DarkSide-20k TPC and relevant optical models for light propagation.

The electroluminescence signal (S2) is simulated as isotropically distributed photons with the vacuum-ultraviolet (VUV) wavelength of $128 \mathrm{~nm}$ originating in a thin layer of gas argon at the top of an octagonal TPC with the planar dimensions from one edge of the TPC to the other of $\sim 3.5 \mathrm{~m}$. This light is propagated in the detector volume and is eventually wavelengthshifted to $420 \mathrm{~nm}$ in a Tetraphenyl Butadiene (TPB) layer covering the transparent acrylic anode, and is subsequently detected by an array of 4100 silicon photodetection modules of $\sim 5 \times 5 \mathrm{~cm}^{2}$ (figure 1 ).

Only the fraction of light detected by the top photosensor array is of interest to the present study, and the bottom fraction of the electroluminescence signal is ignored, as light arrives with a large solid angle due to the TPC height and after multiple scattering. The illumination is not localized and additional information does not benefit the reconstruction. However, it can be noted that information from both arrays can be used for more complex studies, such as detection of anomalies and signal quality checks on real data.

The performed studies take into account scenarios of partial loss of photosensors at the level of a single module and a full mechanical unit, as well as signal non-linearity and channel saturation at various levels, and granularity of the top photodetection array.

Three different algorithms have been developed and are described in the following sections.

\subsection{Fully Connected Layers}

Fully Connected (FC) layers are used among a large variety of applications in the machine learning realm due to the lack of manipulation the data needs before being fed to a model [14]. This allows for the network to learn features present in the raw data given at hand with relative ease. However, for large amounts of input dimensions space, this simplicity comes at the expense of requiring many parameters, leading to computationally expensive processes. Nonetheless, this method was used to investigate the performance of using exclusively a DNN of FC layers. The model in figure 4 describes the architecture used for this approach; it is composed of the input layer, three hidden layers with dropout and regularization included to prevent over-fitting, and a final output layer with two output neurons that correspond to $x$ and $y$ position estimates.

\subsection{Convolutional Networks}

\subsubsection{Conv1D}

Other standard machine learning structures are 1D CNNs. The foundation of these assemblies is scalar multiplication and addition, making them widely used among a variety of applications parting from the fact that they are low cost and perform in real-time [15]. 1D Convolution methods can be used to extract data features [16] and are widely used as a preceding block in general machine learning (ML) models.

With the purpose of position reconstruction, this methodology was also explored by building the model shown in figure 5. This structure is composed of the block which performs the one-dimensional convolution on the input data and a block that contains a DNN of FC layers. As the data are fed forward in the convolutions block, the filter size of the convolution is kept 


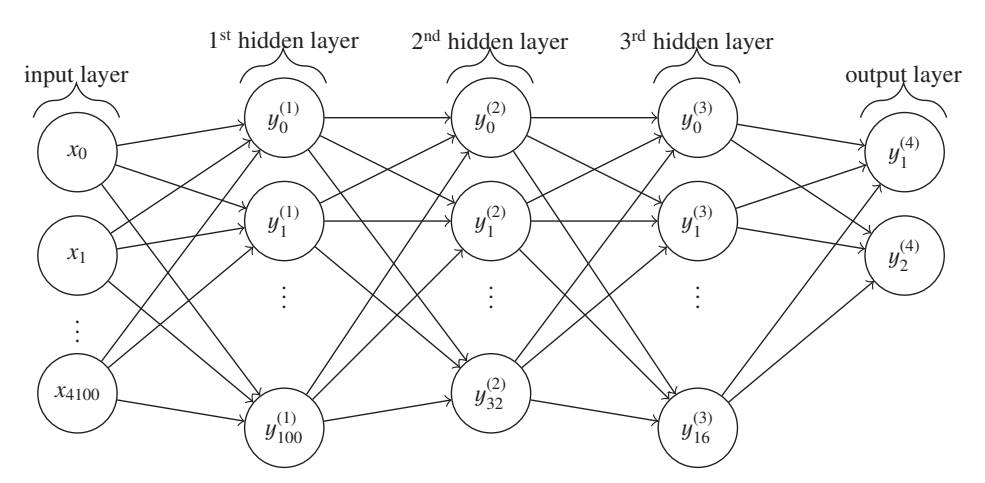

Figure 4. Network graph of a 4 layer DNN with 4100 inputs and 2 outputs used for the method described in section 2.1 .

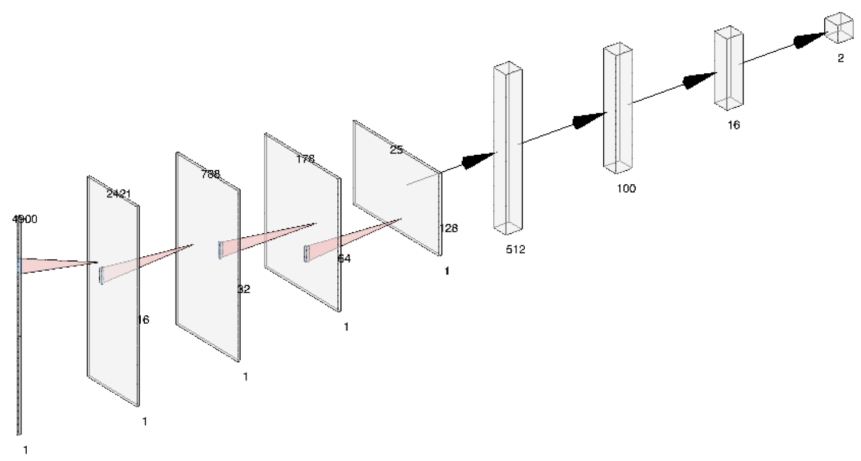

Figure 5. Architecture of the 1D CNN trained for the XY reconstruction in the DarkSide-20k experiment with 4100 photosensors in the top array.

constant while the feature space is increased. Furthermore, pooling layers are used in between hidden layers to reduce the dimensionality of the parameter space before reaching the following block. Once the convolution sector is finished, the data are flattened into a single dimension array and fed to the DNN which outputs the reconstructed positions. The main advantage of this approach over strictly using a DNN is that by the time the first layer in the FC network is reached, the number of dimensions has been largely reduced and the parameter toll is approximately one order of magnitude smaller than in the previous methodology.

\subsubsection{Conv2D}

Similar to its one-dimensional counterpart, 2D convolution is also widely used as a preceding structure to DNNs in a variety of applications, for instance, picture recognition [17]. These structures rely upon feature extraction and classification processes and are more conveniently used for data structures that are two-dimensional in nature. One of their major advantages is the facility to adapt these architectures to different sizes as well as being immutable to data translations and scaling [15]. This property is widely exploited in section 3 in this study. 


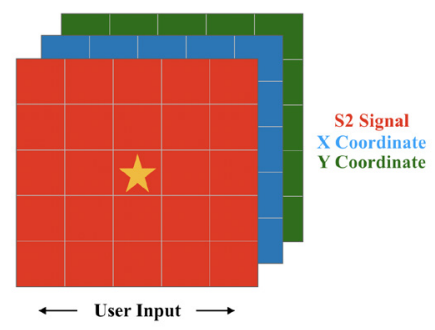

(a)

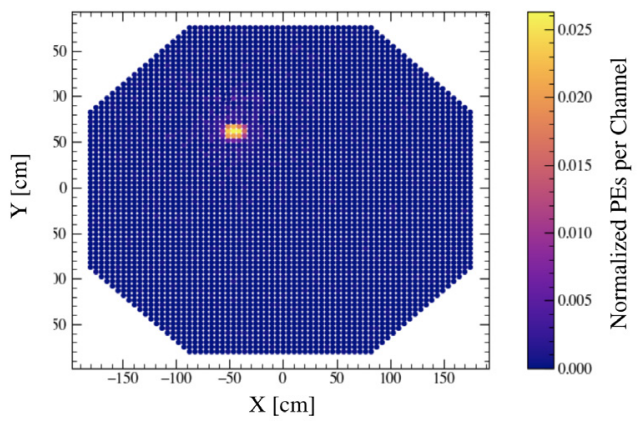

(b)

Figure 6. The architecture of the 2D CNN optimized for the XY reconstruction in the DarkSide-20k experiment: a) Model Input data format. b) An example of the light fraction map generated with the optical model implemented in G4DS. The top photosensor array consists of 4100 photosensors of $\sim 5 \times 5 \mathrm{~cm}^{2}$ size.

For this method, the user can specify the input number of photosensors around the channel that collects most of the light. A three-level deep square size array based on the user's input (figure 6) is filled with the light and position information of each channel before feeding it to the model. Similar to the methodology described in the previous section, after the 2D convolution block, the data is flattened and fed into the DNN of FC layers and the event's position is reconstructed.

\section{Performance Evaluation}

This section describes the performance of the three different approaches used for position reconstruction. Furthermore, the method described in section 2.2.2 is used to investigate the detector response when affected by saturation or loss of photosensor channels. Table 3 shows the spatial resolution for each of the methods applied. The spatial resolution is defined as the standard deviation of the reconstructed error distribution of each coordinate. The reconstructed error is simply the difference between the MC positions and the reconstructed position outputted by the model. The FC DNN for instance, are $30 \%$ better when reconstructing low energy events, [10-3000] photoelectrons, with respect to more traditional methods like Center of Gravity (CoG) developed for the DarkSide-20k detector.

\begin{tabular}{|c|c|c|}
\hline Method & $\sigma_{x}[\mathrm{~cm}]$ & $\sigma_{y}[\mathrm{~cm}]$ \\
\hline FC DNN & 0.34 & 0.33 \\
1D Convolution & 0.53 & 0.54 \\
2D Convolution & 0.55 & 0.53 \\
\hline
\end{tabular}

Table 1. Spatial resolution obtained with the different methodologies used for XY position reconstruction for events with [10-10000] photoelectrons.

Due to edge effects like wall reflections and irregular light patterns, the spatial resolution tends to worsen as the radial distance $(R)$ from the center of the detector increases. Figure 7 illustrates the performance of the model as a function of radial distance. The increasing 
trend is clearly observed for $R>160 \mathrm{~cm}$, with the spatial resolution reaching values of $\sigma_{x, y} \sim 1 \mathrm{~cm}$. Although this is a noticeable increase in the spatial resolution, CoG methods developed for the DarkSide-20k detector consistently show spatial resolutions of $\sigma_{x, y}>2 \mathrm{~cm}$ for radial distances of the same range.
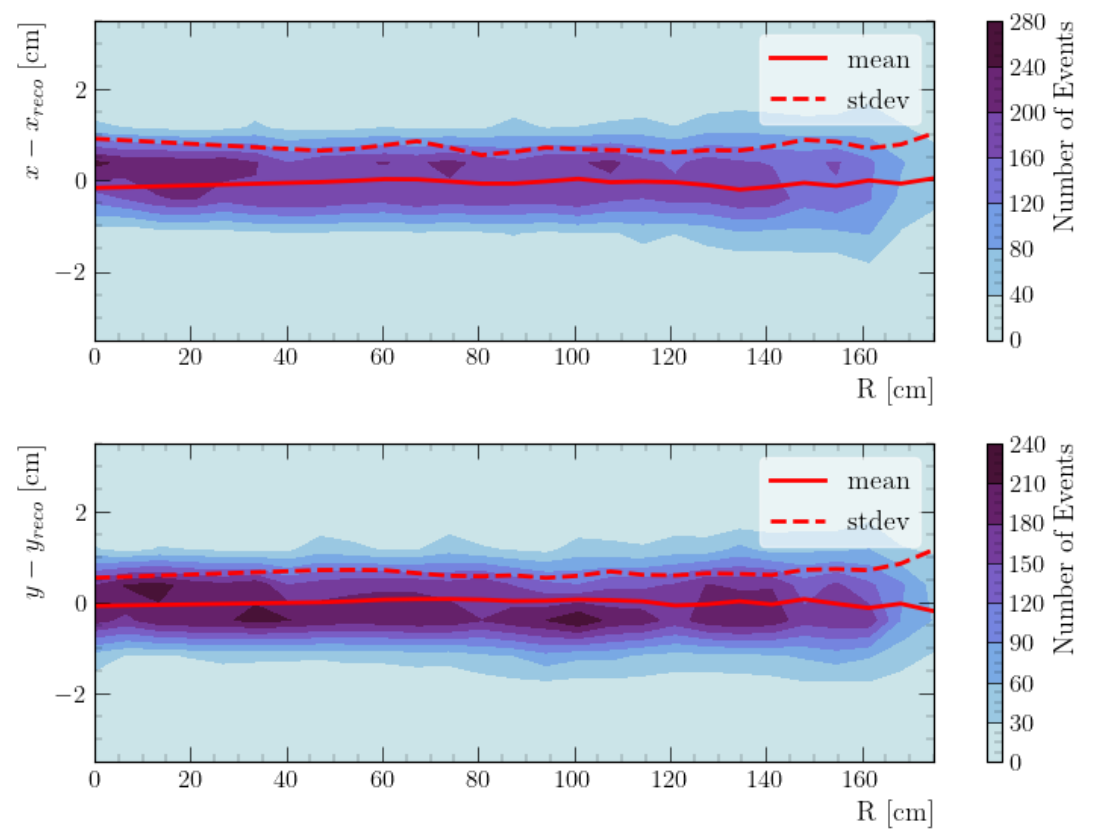

Figure 7. Distribution of the spatial resolution as a function of the radial distance for the $x$ and $y$ coordinates.

Moreover, the energy dependence of the spatial resolution is also evaluated and shown in figure 8 . This is done by extracting the mean and standard deviation from the distributions of the reconstructed error as a function of the number of photoelectrons (NPE) detected per event. Due to the lack of a well defined light pattern in the photosensor array for low energy events, these are reconstructed more poorly, reaching values of $\sigma_{x} \sim 2.8 \mathrm{~cm}$ and $\sigma_{y} \sim 2.5 \mathrm{~cm}$ for events under $500 \mathrm{NPE}$.

\subsection{Response to the Loss of Photosensor Channels}

The DarkSide-20k detector is expected to have a run-life in the order of 5 years after the commissioning phase [12]. Due to this long operational period, some channels could experience a failure during the life of the experiment. This will to a certain measure affect the XY spatial resolution since a definite number of photosensors will not be able to register any light information.

With this purpose of quantifying this effect, the spatial resolution was studied for a different number of inactive channels. These channels were randomly distributed throughout the full top array of the detector. Any signal that was registered in a channel that was characterized as invalid was set to 0 . A model previously trained with the full functioning array was used for reconstruction. For each configuration of invalid channels, the reconstruction error distribution was computed and the spatial resolution was evaluated. The results are shown in figure 9 

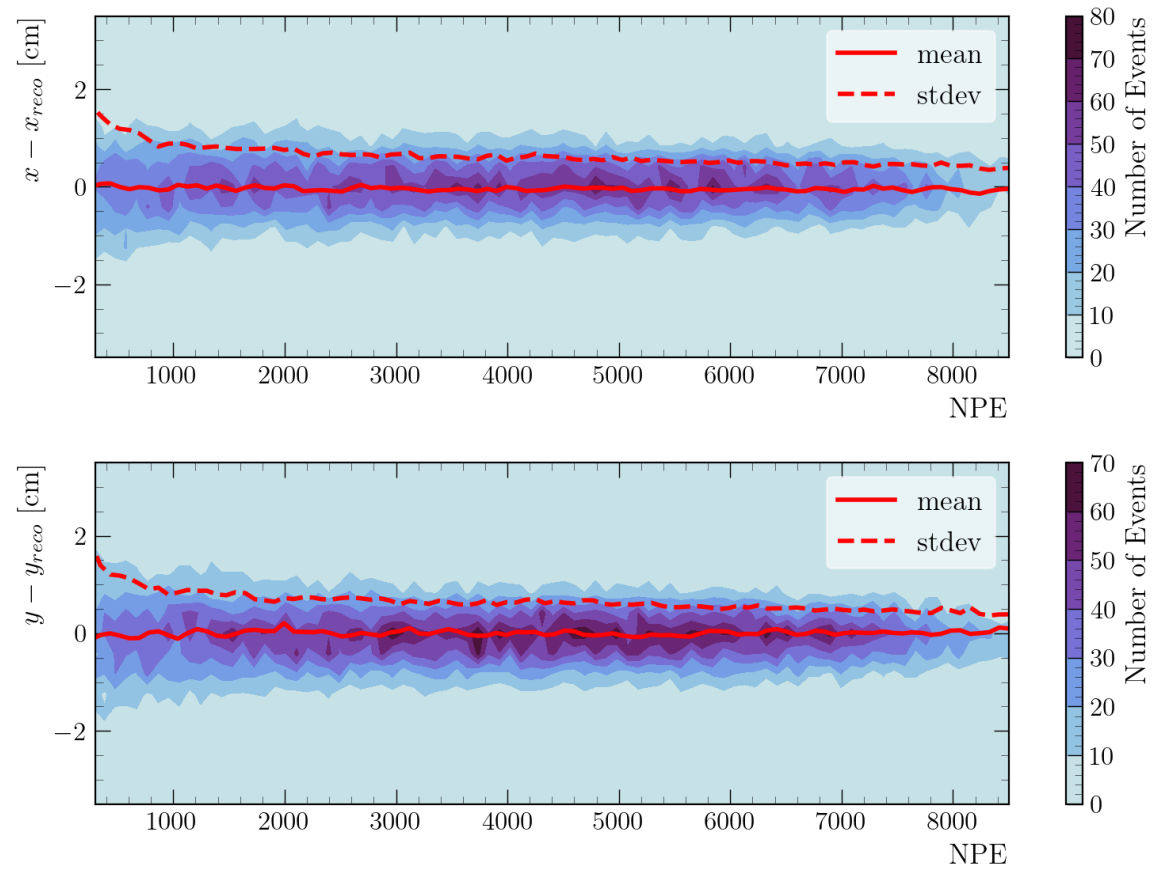

Figure 8. Distribution of the spatial resolution as a function of NPE for the $\mathrm{X}$ and $\mathrm{Y}$ coordinates.

a); the spatial resolution increases linearly as the number of inactive photosensors. This was done with the 2D convolution methodology, which adequately performs for a low number of inactive photosensors since most of the top array channels are ignored for the reconstruction. It is only above 200 inactive channels that the spatial reconstruction starts to increase above levels of $\sigma_{r}>1 \mathrm{~cm}$

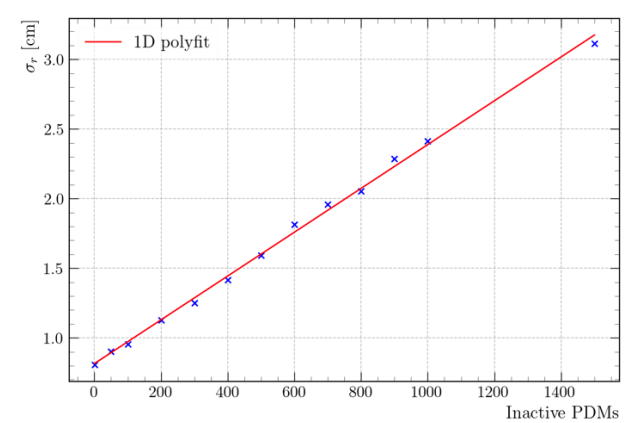

(a)

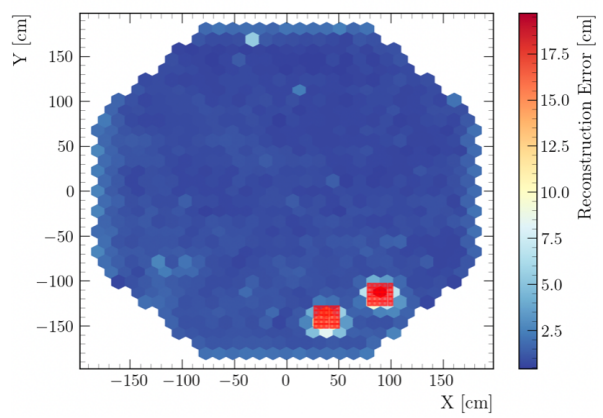

(b)

Figure 9. a) Spatial Resolution as a function of number of inactive channels. b) Error map of the detector with $25 \times 5$ groups of inactive photosensors. 
On a different note, the photosensors of the DarkSide-20k experiment will be grouped in $5 \times 5$ channel arrays forming a photodetection unit (PDU). The power modules for these photosensors will be jointly connected such that there is a potential risk of a full PDU malfunctioning. This would imply the loss of 25 channels in a localized region, which largely affects the position reconstruction capabilities of the experiment. Figure 9 b) shows an error map of the detector in which two PDUs have been marked as inactive. The reconstruction error in the local $5 \times 5$ photosensor region increases up to levels above $17 \mathrm{~cm}$.

\subsection{Signal Saturation Effects}

Another physical phenomenon to consider when reconstructing the position of a given event is the signal non-linearity. Due to the photosenors employed in the experiment, the specifics of the electronics and signal readout systems, and the fact that direct dark matter experiments are tuned for very low energy signals and thresholds, saturation effects in single modules could be observed for signals of $\sim 150$ NPEs / PDM.

Unlike inactive channels where all signal from the sensor is lost, saturation limits the maximum NPEs that a channel can register, which can drastically change the shape of the S2 distribution in the XY plane. Since the reconstruction of the event position depends on the NPEs recorded per channel, only the channels that are saturated and the maximum amount of PEs registered are of interest. To investigate this effect, events with different NPEs were reconstructed while changing the saturation threshold per channel. Figure 10 shows the correlation between NPEs at which channels can be saturated and the corresponding spatial resolution. Since higher energy events are usually reconstructed better, it is important to note that when accounting for saturation these will tend to be reconstructed poorly especially for low values of the saturation threshold. According to this figure, saturation thresholds below 200 PEs will rapidly start increasing the spatial resolution.

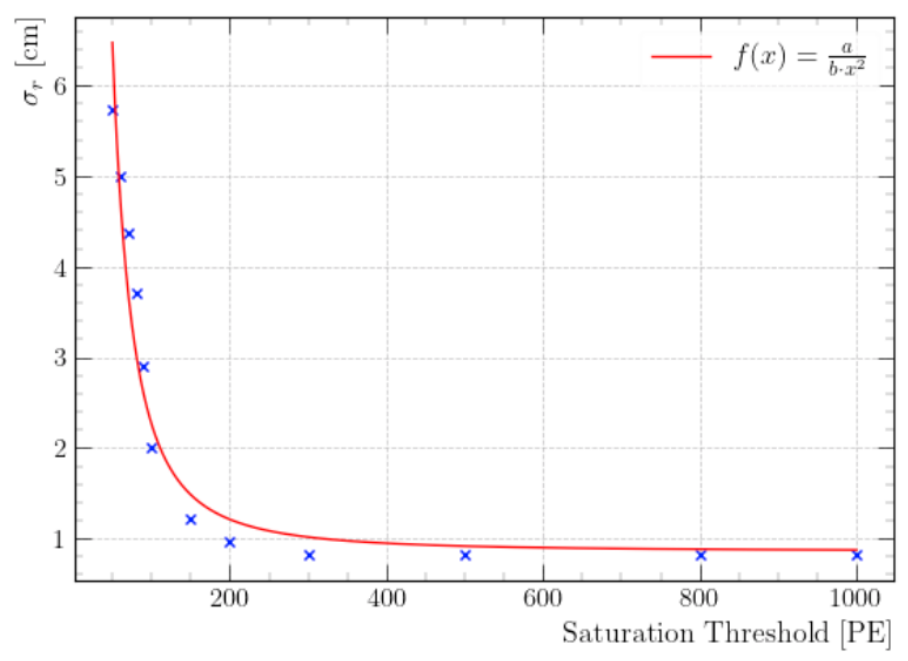

Figure 10. Spatial Resolution as a function of the saturation threshold. 


\section{Conclusions}

A software package was developed for event vertex XY reconstruction in dual-phase TPCs, based on modern deep learning methods. Network training, testing, and validation were performed on the data simulated with a model of the DarkSide-20k TPC with unprecedented results.

The XY resolution achieved with the deep learning techniques matches well the design value of $1 / 10$ th of the photosensor size. It is observed to be even better with the FC DNN and is a subject of further investigation. As this type of neural network uses all information from the entire photosensor array and performs well despite a large number of training parameters, it will be studied for other applications complementary to vertex reconstruction, such as target fiducialization and discrimination of single and multi-it events.

The developed package includes the necessary modularity and options to be easily adopted for TPCs with the different size, shape, and photosensor array granularity, with the specifics of the detector effects and light propagation to be taken into account in the dedicated Monte Carlo simulations.

Particularly interesting for the next steps is hardware (GPU [18] or FPGA-based [19]) acceleration for deep learning inference within the data reconstruction workflow, and hence possibilities of a coarse online event vertex reconstruction for trigger purposes, to reduce the data bandwidth and free disk space and computing resources.

\section{References}

[1] V. N. Solovov et al. (ZEPLIN-III Collaboration), IEEE Transactions on Nuclear Science 59, 6 (2012), 112 . 1481

[2] E. Aprile et al. (XENON Collaboration), Astroparticle Physics 35, 573 (2012), 1107.2155

[3] C. Adams, M. Alrashed, R. An, J. Anthony, J. Asaadi, A. Ashkenazi, M. Auger, S. Balasubramanian, B. Baller, C. Barnes et al., Physical Review D 99 (2019)

[4] J. Strube, K. Bhattacharya, E. Church, J. Daily, S. Malachi, S. Charles, W. Kevin, EPJ Web of Conferences 214, 06016 (2019)

[5] J. Liu, J. Ott, J. Collado, B. Jargowsky, W. Wu, J. Bian, P. Baldi, Deep-learning-based kinematic reconstruction for dune (2020), 2012.06181

[6] M. Wang, T. Yang, M.A. Flechas, P. Harris, B. Hawks, B. Holzman, K. Knoepfel, J. Krupa, K. Pedro, N. Tran, Gpu-accelerated machine learning inference as a service for computing in neutrino experiments (2020), 2009.04509

[7] S. Delaquis, M. Jewell, I. Ostrovskiy, M. Weber, T. Ziegler, J. Dalmasson, L. Kaufman, T. Richards, J. Albert, G. Anton et al., Journal of Instrumentation 13, P08023-P08023 (2018)

[8] C.K. Khosa, L. Mars, J. Richards, V. Sanz, Journal of Physics G: Nuclear and Particle Physics 47, 095201 (2020)

[9] A.I. A. Grobov, Convolutional Neural Network Approach to Event Position Reconstruction in DarkSide-50 Experiment, in Journal of Physics: Conference Series. Vol. 1690, 5th International Conference on Particle Physics and Astrophysics at MEPhI (2020)

[10] S. Delaquis, M. Jewell, I. Ostrovskiy, M. Weber, T. Ziegler, J. Dalmasson, L. Kaufman, T. Richards, J. Albert, G. Anton et al., Journal of Instrumentation 13, P08023-P08023 (2018)

[11] P. Agnes et al. (DarkSide collaboration) (2020), 2011.07819 
[12] C. Aalseth et al. (DarkSide collaboration), Eur. Phys. J. Plus 133, 131 (2018), 1707.08145

[13] P. Agnes et al. (DarkSide collaboration), JINST 12, 10015 (2017), 1707.056309

[14] S.S. Basha, S.R. Dubey, V. Pulabaigari, S. Mukherjee, Neurocomputing 378, 112 (2020)

[15] S. Kiranyaz, O. Avci, O. Abdeljaber, T. Ince, M. Gabbouj, D.J. Inman, Mechanical Systems and Signal Processing 151, 107398 (2021)

[16] L. Zhong, L. Hu, H. Zhou, Remote Sensing of Environment 221, 430 (2019)

[17] F. Sultana, A. Sufian, P. Dutta, 2018 Fourth International Conference on Research in Computational Intelligence and Communication Networks (ICRCICN) (2018)

[18] J. Krupa et al. (2020), 2007. 10359

[19] J. Duarte, S. Han, P. Harris, S. Jindariani, E. Kreinar, B. Kreis, J. Ngadiuba, M. Pierini, R. Rivera, N. Tran et al., Journal of Instrumentation 13, P07027-P07027 (2018) 